317 | juillet-septembre 1999

Numéro spécial : France-Allemagne. Interactions, références

\title{
La Guyane sous la Révolution, ou l'impasse de la révolution pacifique
}

\section{Bernard Gainot}

\section{(2) OpenEdition \\ Journals}

Édition électronique

URL : https://journals.openedition.org/ahrf/923

DOI : 10.4000/ahrf.923

ISSN : 1952-403X

Éditeur :

Armand Colin, Société des études robespierristes

Édition imprimée

Date de publication : 1 septembre 1999

Pagination : 550-552

ISSN : 0003-4436

Référence électronique

Bernard Gainot, «La Guyane sous la Révolution, ou l'impasse de la révolution pacifique », Annales historiques de la Révolution française [En ligne], 317 | juillet-septembre 1999, mis en ligne le 10 avril 2006, consulté le 23 avril 2022. URL : http://journals.openedition.org/ahrf/923 ; DOI : https://doi.org/ 10.4000/ahrf.923

Ce document a été généré automatiquement le 23 avril 2022.

Tous droits réservés 


\title{
La Guyane sous la Révolution, ou l'impasse de la révolution pacifique
}

\author{
Bernard Gainot
}

\section{RÉFÉRENCE}

Yves BENOT, La Guyane sous la Révolution, ou l'impasse de la révolution pacifique (Ibis rouge Éditions, Kourou, 1997), sous le patronage du programme Route de l'esclave de l'UNESCO, 222 pp., $145 \mathrm{~F}$

1 Cette synthèse comble une lacune concernant l'histoire de la plus méconnue des colonies de la République. Le dernier ouvrage d'Yves Bénot, à l'instar de ses précédentes mises au point sur les problèmes coloniaux de la période révolutionnaire et impériale, se place d'emblée à contre-courant de l'historiographie dominante concernant la première abolition de l'esclavage. Détracteurs des idéaux révolutionnaires et apologistes $d u$ volontarisme communautaire, voire racial, se retrouvent d'accord sur un point: le décret d'abolition du 16 pluviôse an II est un rideau de fumée qui n'a abusé que quelques naïfs philanthropes métropolitains. À travers un dépouillement particulièrement soigné des archives, l'auteur s'efforce de prendre en compte les réalités juridiques et les enjeux sociaux de la période abolitionniste autrement que par le discours incantatoire.

2 L'évolution de Saint-Domingue est généralement emblématique des formes et des enjeux de cette première période abolitionniste; la Guadeloupe est connue grâce aux travaux de Anne Pérotin-Dumont et Jacques Adélaïde-Merlande, notamment; nous disposons désormais de l'ouvrage de Claude Wanquet sur les Mascareignes. Mais la Guyane? La singularité de cette colonie vient du fait que le décret d'abolition n'est pas le résultat d'une insurrection des esclaves en armes, d'une reconquête militaire sous la conduite d'officiers noirs et métis, mais l'acte délibéré du représentant du pouvoir métropolitain: une abolition volontariste, donc un processus pacifique. Révolution pacifique: l'association renvoie-t-elle pour autant à la notion de révolution passive? Les 
nombreux soulèvements de populations serviles ou des nouveaux libres, les résistances de la quasi-totalité des propriétaires d'origine européenne, prouveraient plutôt le contraire. Yves Bénot impute cette méconnaissance de la réalité des soulèvements serviles (comme celui de l'Approuague en décembre 1790) à l'analphabétisme; en 1789, il y a 11000 esclaves pour 1300 Blancs, mais un seul esclave qui sache lire et écrire; Hector Menénins, élu en 1794, exécuté en 1796. La proportion est plus élevée dans les autres territoires des Indes occidentales.

3 La période abolitionniste proprement dite est marquée par la forte personnalité des deux représentants de la métropole: Jeannet-Oudin et Cointet. Le premier est le cousin de Danton: il est gouverneur du 14 avril 1793 à novembre 1794; puis il revient comme agent d'avril 1796 à novembre 1798. Dans l'intervalle, il a confié le pouvoir à un militaire jacobin, Cointet. Tous deux sont des abolitionnistes convaincus; il y en avait un certain nombre dans le personnel politique de la Première République, Directoire compris. Il n'est pas inutile de le rappeler et d'insister sur ce point aujourd'hui.

4 Le décret du 16 pluviôse est arrivé à Cayenne sans accompagnement d'instructions concernant ses modalités d'application. Nous voici au cœur de la problématique qui sous-tend cette étude; quelle organisation économique et sociale pour garantir et asseoir solidement le régime juridique de la liberté générale? L'absence d'instructions contraint les autorités de Cayenne à innover.

5 Le travail sur les plantations fait l'objet de contrats négociés entre les maîtres européens et les cultivateurs «nouveaux libres». Ces contrats de travail matérialisent la conception républicaine de l'Égalité, fondée sur l'union de toutes les couleurs. De même, tous les Noirs deviennent citoyens, et obtiennent le droit de suffrage. Les «nouveaux libres» sont admis au Club de Cayenne: dans une grande liesse populaire, le 14 juillet 1794 est l'apogée de cette Révolution pacifique.

6 Mais une fraction significative des «nouveaux libres» et ce, en dépit des interdictions formelles, va s'établir sur des exploitations familiales, et produire, non pas exclusivement dans un cadre auto-suffisant, mais aussi en vue de dégager un petit surplus commercialisable.

7 Examinant les statistiques à l'exportation après l'abolition, Bénot balaye les arguments qui entretiennent le mythe de l'effondrement total de l'économie imputable au régime de la liberté générale. Mieux, il démontre que les faibles excédents familiaux ont évité la famine généralisée vers laquelle se dirigeait inéluctablement la colonie du fait du blocus maritime.

8 L'autre réalité guyanaise est sans doute mieux connue des historiens de la décennie révolutionnaire; c'est la "guillotine sèche», qui reçoit pêle-mêle les grands coupables terroristes de l'an III (Billaud-Varenne et Collot d'Herbois) et les déportés royalistes après le 18 fructidor an V; tout en rappelant que les victimes les plus nombreuses de ces déportations furent les prêtres réfractaires sous le Directoire. Le grand mérite de l'ouvrage d'Yves Bénot est de modifier la perspective, de nous parler des déportations depuis l'«autre rive», de croiser les convulsions de l'État métropolitain, et les incertitudes du destin des colonies.

9 La Guyane comme «terre de transportation» est une vieille idée; pour les déportés, c'est un lieu de relégation, et non un camp de concentration. Théoriquement, ils ne peuvent toutefois résider à Cayenne. Le gouverneur Cointet reçut les anciens membres des comités; selon, Bénot, c'est Collot qui fut le plus ouvert aux réalités sociopolitiques de 
la colonie. Il exerça une certaine influence parmi les troupes, et les planteurs blancs, qui lui étaient très hostiles, virent cette influence dans les troubles de pluviôse an IV (janvier 1796). Quelques postes militaires furent attaqués, il y eut une quinzaine d'exécutions, dont celle d'Hector Ménénius. L'auteur, au terme d'une minutieuse analyse des rares pièces disponibles, considère que le but de cette insurrection pouvait être l'indépendance d'une Guyane noire.

10 L'influence des déportés «fructidorisés» s'exerça dans une toute autre direction; la plupart d'entre eux avaient participé, dans les Conseils de l'an V, à la tentative d'annulation du décret du 16 pluviôse. Ils tissent des liens étroits avec le milieu des planteurs blancs, nostalgiques de l'ancien ordre esclavagiste, prépondérants dans les autorités départementales. Tous passent leur temps à dénoncer l'action de Cointet, puis de Jeannet. Tous participent au coup d'État contre l'agent du Directoire Burnel, qui a remplacé Jeannet en novembre 1798. Ce coup d'État, qui eut pour conséquence le rembarquement de Burnel, et la remise du pouvoir dans les mains d'un gouvernement provisoire dominé par les colons, eut lieu à la date symbolique du 19 brumaire an VIII! Dès lors, tout était en place pour le dénouement de cette phase ambiguë de la première abolition; Victor Hugues, nommé par le Directoire, arrive à Cayenne le 8 janvier 1800 . Les réglements de culture qu'il édicte, assignant la main-d'œuvre noire à résidence, sont le prélude au rétablissement de l'esclavage de la fin 1802.

11 Le prétexte au coup d'État contre Burnel avait été sa tentative de lever un second bataillon noir (le premier l'ayant été levé à l'initiative de Cointet, en germinal an III, et c'était là le grief principal des Blancs envers l'action du gouverneur). Yves Bénot rappelle aussi qu'un certain nombre de mesures furent prises dans le cadre de la départementalisation, régime d'égalité juridique mis en place par la loi de janvier 1798; une école fut créée à Cayenne, un corps de gendarmerie fut organisé, composé de Noirs et de métis.

12 Mais le cœur du problème fut bien la question de l'armement des «nouveaux libres». Si le bataillon noir se montra loyal envers les autorités républicaines, permettant à ces dernières de réprimer les tentatives d'insurrection, l'auteur insiste en fin d'ouvrage sur la généralisation des maquis qui persistèrent de la restauration de l'ordre esclavagiste à l'occupation Portugaise de 1809. Un fait qui contredit l'analyse du processus abolitionniste à la Guyane comme révolution passive, et qui invite à revoir toute la période avec un regard neuf, à l'envisager comme période d'expérimentation. Et à approfondir les recherches de terrain à la lumière des problèmes posés par cet ouvrage essentiel pour l'histoire des colonies entre 1789 et 1815. 